\title{
EDGE-DISJOINT HOMOTOPIC PATHS IN STRAIGHT-LINE PLANAR GRAPHS*
}

\author{
A. SCHRIJVER $\dagger$
}

\begin{abstract}
Let $G$ be a planar graph, embedded without crossings in the euclidean plane $\mathbb{R}^{2}$, and let $I_{1}, \cdots$, $I_{p}$ be some of its faces (including the unbounded face), considered as open sets. Suppose there exist (straight) line segments $L_{1}, \cdots, L_{t}$ in $\mathbb{R}^{2}$ so that $G \cup I_{1} \cup \cdots \cup I_{p}=L_{1} \cup \cdots \cup L_{t} \cup I_{1} \cup \cdots \cup I_{p}$ and so that each $L_{i}$ has its end points in $I_{1} \cup \cdots \cup I_{p}$. Let $C_{1}, \cdots, C_{k}$ be curves in $\mathbb{R}^{2} \backslash\left(I_{1} \cup \cdots \cup I_{p}\right)$ with end points in vertices of $G$. Conditions are described under which there exist pairwise edge-disjoint paths $P_{1}, \cdots, P_{k}$ in $G$ so that $P_{i}$ is homotopic to $C_{i}$ in $\mathbb{R}^{2} \backslash\left(I_{1} \cup \cdots \cup I_{p}\right)$, for $i=1, \cdots, k$. This extends results of Kaufmann and Mehlhorn for graphs derived from the rectangular grid.
\end{abstract}

Key words. edge-disjoint, paths, homotopic, packing, planar

AMS(MOS) subject classifications. $05 \mathrm{C} 10,05 \mathrm{C} 38$

1. Introduction and statement of the theorem. Let $G=(V, E)$ be a planar graph, embedded without crossing edges in the euclidean plane $\mathbb{R}^{2}$. We identify $G$ with its image in $\mathbb{R}^{2}$. Let $I_{1}, \cdots, I_{p}$ be some of its faces, including the unbounded face, called the black holes. (We consider faces as open sets.) Moreover, let paths $C_{1}, \cdots, C_{k}$ be given with end points in $V$, not intersecting any black hole. (That is, for each $i, C_{i}$ is a continuous function $[0,1] \rightarrow \mathbb{R}^{2} \backslash\left(I_{1} \cup \cdots \cup I_{p}\right)$ with $C(0), C(1) \in V$.)

Motivated by the automatic design of integrated circuits, Mehlhorn posed the following question:

Under which conditions do there exist pairwise edge-disjoint paths $P_{1}, \cdots$, $P_{k}$ in $G$ so that $P_{i}$ is homotopic to $C_{i}$ in the space $\mathbb{R}^{2} \backslash\left(I_{1} \cup \cdots \cup I_{p}\right)$ (for $i=1$, $\cdots, k)$ ?

Here a path in $G$ is a continuous function $P:[0,1] \rightarrow G$ with $P(0), P(1) \in V$. Paths $P_{1}, \cdots, P_{k}$ are pairwise edge-disjoint if the following holds: if $P_{i}(x)=P_{j}(y) \notin V$ then $x=y$ and $i=j$. (In particular, if $P_{1}, \cdots, P_{k}$ are pairwise edge-disjoint, then each $P_{i}$ does not pass the same edge more than once.) Two paths $P, C:[0,1] \rightarrow \mathbb{R}^{2} \backslash$ $\left(I_{1} \cup \cdots \cup I_{p}\right)$ are homotopic (in $\mathbb{R}^{2} \backslash\left(I_{1} \cup \cdots \cup I_{p}\right)$ ), denoted by $P \sim C$, if there exists a continuous function $\Phi:[0,1] \times[0,1] \rightarrow \mathbb{R}^{2} \backslash\left(I_{1} \cup \cdots \cup I_{p}\right)$ so that for all $x \in$ $[0,1]: \Phi(x, 0)=P(x), \Phi(x, 1)=C(x), \Phi(0, x)=P(0), \Phi(1, x)=P(1)$. (In particular, $P(0)=C(0)$ and $P(1)=C(1)$.)

Mehlhorn proposed to study question (1) with the help of the following "cuts." A (homotopic) cut is a continuous function $D:[0,1] \rightarrow \mathbb{R}^{2} \backslash\left(V \cup I_{1} \cup \cdots \cup I_{p}\right)$ so that $D(0)$ and $D(1)$ belong to the boundary of $I_{1} \cup \cdots \cup I_{p}$ and so that $\left|D^{-1}(G)\right|$ is finite. The cut condition ( for $G ; I_{1}, \cdots, I_{p} ; C_{1}, \cdots, C_{k}$ ) is:

$$
\text { (cut condition) for each cut } D: \operatorname{cr}(G, D) \geqq \sum_{i=1}^{k} \operatorname{mincr}\left(C_{i}, D\right) \text {. }
$$

\footnotetext{
* Received by the editors August 10,1987; accepted for publication (in revised form) December 6, 1988.

$\dagger$ Mathematical Centre, Kruislaan 413, 1098 SJ Amsterdam, the Netherlands.
} 
Here we use the following notation for curves $C, D:[0,1] \rightarrow \mathbb{R}^{2} \backslash\left(I_{1} \cup \cdots \cup I_{p}\right)$ :

$$
\begin{aligned}
& \operatorname{cr}(G, D):=|\{y \in[0,1] \mid D(y) \in G\}|, \\
& \operatorname{cr}(C, D):=|\{(x, y) \in[0,1] \times[0,1] \mid C(x)=D(y)\}|, \\
& \operatorname{mincr}(C, D):=\min \left\{\operatorname{cr}(\tilde{C}, \tilde{D}) \mid \tilde{C} \sim C, \tilde{D} \sim D \text { in } \mathbb{R}^{2} \backslash\left(I_{1} \cup \cdots \cup I_{p}\right)\right\} .
\end{aligned}
$$

Clearly, the cut condition is a necessary condition for a positive answer to question (1). It is generally not sufficient, not even for quite simple situations. For example, take $k=2, p=1$, and consider

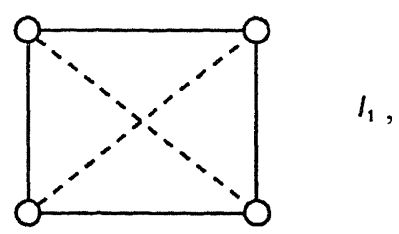

where the straight lines stand for edges of $G$ and where the interrupted lines stand for curves $C_{1}$ and $C_{2}$.

It turned out that one additional condition, the so-called parity condition, can be helpful (cf. $\S 2$ below):

(4) (parity condition) for each cut $D: \operatorname{cr}(G, D) \equiv \sum_{i=1}^{k} \operatorname{mincr}\left(C_{i}, D\right)(\bmod 2)$.

Let us now state our theorem. We say that $G ; I_{1}, \cdots, I_{p} ; C_{1}, \cdots, C_{k}$ is in the straight-line case if

there are line segments $L_{1}, \cdots, L_{t}$ in $\mathbb{R}^{2}$ so that $G \cup I_{1} \cup \cdots \cup I_{p}=L_{1} \cup \cdots$ $\cup L_{t} \cup I_{1} \cup \cdots \cup I_{p}$ and so that each $L_{j}$ has its end points in $I_{1} \cup \cdots \cup I_{p^{\prime}}$

and

if the aperture at vertex $v$ of $G$ is larger than $180^{\circ}$, then the number of times $v$ occurs as end point of the curves $C_{i}$ is not larger than the number of edges terminating at $v$.

Here the aperture at vertex $v$ of $G$ is the largest angle that can be made at $v$ so that none of the black holes adjacent to $v$ intersect the interior of the angle. (More formally, let $\rho>0$ be so that the circle $K$ of radius $\rho$ and centre $v$ does not contain any other vertex of $G$ in its interior and does not intersect any edge except for those adjacent to $v$. Let $K \backslash\left(I_{1} \cup \cdots \cup I_{p}\right)$ have components $K_{1}, \cdots, K_{h}$, making angles $\varphi_{1}, \cdots, \varphi_{h}$. Then the aperture at $v$ is equal to $\max \left\{\varphi_{1}, \cdots, \varphi_{h}\right\}$.) Edge $e=\{(1-\lambda) u+\lambda v \mid 0<\lambda<1\}$ of $G$ is said to terminate at $v$ if for some $\mu>1$ the set $\{(1-\lambda) u+\lambda v \mid 1<\lambda<\mu\}$ is contained in $I_{1} \cup \cdots \cup I_{p}$.

THEOREM. If we are in the straight-line case and the parity condition holds, then there exist pairwise edge-disjoint paths as in (1) if and only if the cut condition holds.

As an illustration, Fig. I gives an example of the straight-line case (where the shaded faces, together with the unbounded face, are the black holes, and where the interrupted curves stand for the paths $C_{i}$ ).

The theorem generalizes a result of Kaufmann and Mehlhorn [2] for graphs derived from the rectangular grid in the following way. $G$ is a finite subgraph of the rectangular grid. (That is, $V$ is a finite subset of $\mathbb{Z}^{2}$ and each edge is a line segment of length 1.) $I_{1}, \cdots, I_{p}$ are exactly those faces of $G$ that are not bounded by exactly four edges of $G$. 


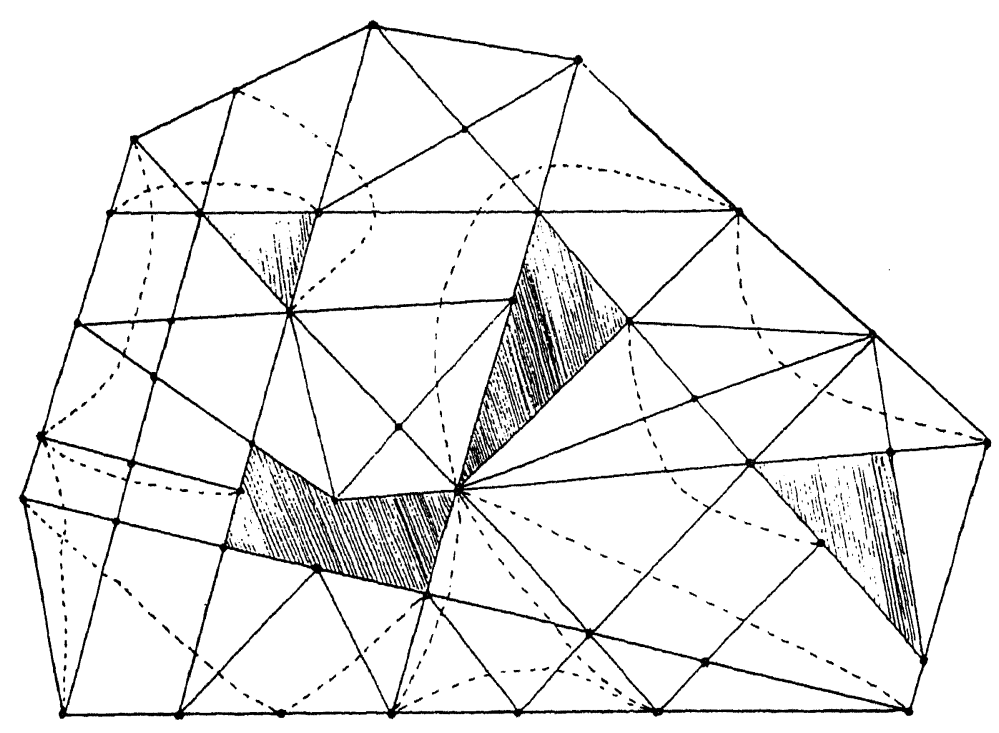

FIG. 1

Moreover, for each vertex $v$ it is required that deg $(v)+r(v) \leqq 4$, where deg $(v)$ denotes the degree of $v$ in $G$, and

$$
r(v):=\left|\left\{i=1, \cdots, k \mid C_{i}(0)=v\right\}\right|+\left|\left\{i=1, \cdots, k \mid C_{i}(1)=v\right\}\right| .
$$

COROLlARY (Kaufmann and Mehlhorn). If the conditions given in the previous paragraph are satisfied and the parity condition holds, then there exist pairwise edgedisjoint paths as in (1) if and only if the cut condition holds.

In fact, Kaufmann and Mehlhorn found a linear-time algorithm to find these paths, if they exist.

In $\S 4$ we give a proof of our theorem. We make use of a lemma to be proved in $\S 3$ (showing that in the straight-line case we may restrict the cut condition to (almost) straight cuts (analogous to the idea of "1-bend cuts" in [2])), and of results of [4] to be reviewed in $\S 2$.

2. Review of preliminary results. In this section we return to the general case of a planar graph $G=(V, E)$ embedded without crossing edges in the Euclidean plane $\mathbb{R}^{2}$, with black holes $I_{1}, \cdots, I_{p}$ (including the unbounded face) and curves $C_{1}, \cdots, C_{k}$. Let each $C_{i}$ have its end points in vertices on the boundary of $I_{1} \cup \cdots \cup I_{p}$.

It was shown by Okamura and Seymour [3] that if $p=1$ the cut condition together with the parity condition imply the existence of paths as in (1). (Note that for $p=1$ two paths $P, P^{\prime}$ are homotopic if and only if $P(0)=P^{\prime}(0)$ and $P(1)=P^{\prime}(1)$.) This was extended by van Hoesel and Schrijver [1] to $p=2$. It cannot be extended to higher $p$, as is shown for $p=3$ by:

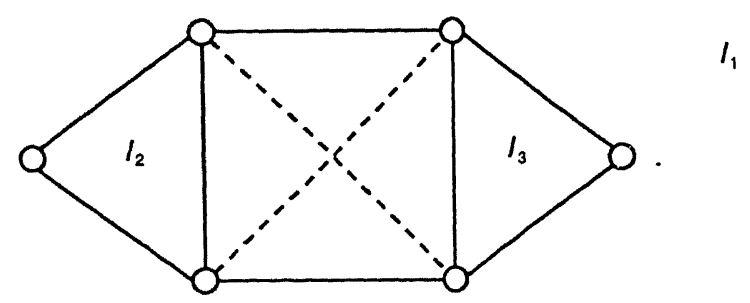


However, it was shown in [4] that, for arbitrary $p$, the cut condition is equivalent to the existence of a "fractional" packing of paths as required, i.e., to the existence of paths $P_{1}^{1}, \cdots, P_{1}^{t_{1}}, P_{2}^{1}, \cdots, P_{k}^{1}, \cdots, P_{k}^{t_{k}}$ and rationals $\lambda_{1}^{1}, \cdots, \lambda_{1}^{t_{1}}, \lambda_{2}^{1}, \cdots, \lambda_{k}^{1}, \cdots$, $\lambda_{k}^{t_{k}}>0$ such that:

$$
\begin{array}{ll}
\text { (i) } P_{i}^{j} \sim C_{i} & \left(i=1, \cdots, k ; j=1, \cdots, t_{i}\right), \\
\text { (ii) } \sum_{j=1}^{t_{i}} \lambda_{i}^{j}=1 & (i=1, \cdots, k), \\
\text { (iii) } \sum_{i=1}^{k} \sum_{j=1}^{t_{i}} \lambda_{i}^{j} \chi^{P_{i}^{j}}(e) \leqq 1 & (e \in E) .
\end{array}
$$

Here $\chi^{P}(e)$ denotes the number of times path $P$ passes edge $e$.

Another result from [4] to be used below was derived with the theory of simplicial approximations. Let $C, D:[0,1] \rightarrow \mathbb{R}^{2} \backslash\left(I_{1} \cup \cdots \cup I_{p}\right)$ be continuous. Let $C(0)$, $C(1), D(0)$, and $D(1)$ be on the boundary of $I_{1} \cup \cdots \cup I_{p}$, with $\{C(0), C(1)\} \cap$ $\{D(0), D(1)\}=\varnothing$. Let

$$
X:=\{(y, z) \in[0,1] \times[0,1] \mid C(y)=D(z)\}
$$

be finite, where each $(y, z)$ in $X$ gives a crossing of $C$ and $D$. For $y, y^{\prime} \in[0,1]$ let $\left.C\right|_{y} ^{y^{\prime}}$ denote the path from $C(y)$ to $C\left(y^{\prime}\right)$ given by:

$$
\left(\left.C\right|_{y} ^{y^{\prime}}\right)(\lambda):=C\left((1-\lambda) y+\lambda y^{\prime}\right) \text { for } \lambda \in[0,1]
$$

similarly for $D$. Define for $(y, z),\left(y^{\prime}, z^{\prime}\right) \in X$ :

$$
(y, z) \approx\left(y^{\prime}, z^{\prime}\right) \Leftrightarrow\left(C||_{y}^{y^{\prime}}\right) \approx\left(D \mid z^{\prime}\right) \quad \text { in } \mathbb{R}^{2} \backslash\left(I_{1} \cup \cdots \cup I_{p}\right) .
$$

We call the classes of the equivalence relation $\approx$ the classes of intersections of $C$ and $D$. Such a class is called odd if it contains an odd number of elements. Let odd $(C, D)$ denote the number of odd classes of $X$. Then

$$
\operatorname{mincr}(C, D)=\operatorname{odd}(C, D) \text {. }
$$

3. A lemma on straight cuts. We call a cut $D:[0,1] \rightarrow \mathbb{R}^{2} \backslash\left(V \cup I_{1} \cup \cdots \cup I_{p}\right)$ a straight cut if

$$
\text { either (i) } D \text { is linear, }
$$

or (ii) the line segment connecting $D(0)$ and $D(1)$ is contained in $G$, the functions $D \mid\left[0, \frac{1}{2}\right]$ and $D \mid\left[\frac{1}{2}, 1\right]$ are linear, there is no vertex of $G$ contained in the interior of the triangle $D(0) D\left(\frac{1}{2}\right) D(1)$, and no edge is intersected more than once by $D$.

In (ii) we might think of $D$ as being very close to the line segment connecting $D(0)$ and $D(1)$. So a straight cut is determined by its end points, in case (12) (ii) up to "slight" homotopic shifts, which, however, do not change the number of intersections with $G$.

LEMMA. In the straight-line case, the cut condition holds if and only if $\operatorname{cr}(G, D) \geqq \sum_{i=1}^{k} \operatorname{mincr}\left(C_{i}, D\right)$ for each straight cut $D$.

Proof. Necessity being trivial, we show sufficiency. Let the cut inequality be satisfied by each straight cut. Suppose there exists a cut $D:[0,1] \rightarrow \mathbb{R}^{2} \backslash\left(V \cup I_{1} \cup \cdots \cup I_{p}\right)$ so that

$$
\operatorname{cr}(G, D)<\sum_{i=1}^{k} \operatorname{mincr}\left(C_{i}, D\right) \text {. }
$$


We choose $D$ satisfying (13) so that $t:=\operatorname{cr}(G, D)$ is as small as possible. The idea of the proof is to straighten out $D$ as much as possible.

First observe that we may assume that if $D(1)$ is not on the line through the edge containing $D(0)$, then the line segment $\overline{D(0) D(1)}$ does not intersect $V$ (this can be achieved by slightly shifting $D(0)$ along the edge containing $D(0)$ ). Moreover, we may assume that there exists an $\varepsilon>0$ so that

(i) $D \mid[0, \varepsilon]$ is linear;

(ii) for all $\delta \in(0, \varepsilon]: D(\delta)$ does not belong to any line through any pair of vertices of $G$ nor to any line through a pair of points consisting of a vertex of $G$ and an intersection of $D$ and $G$.

Let $\lambda_{1}, \cdots, \lambda_{t}$ be so that $0=\lambda_{1}<\lambda_{2}<\cdots<\lambda_{t-1}<\lambda_{t}=1$, with $D\left(\lambda_{i}\right) \in G$ for all $i$. Define

$$
\begin{aligned}
& p_{1}:=D(\varepsilon), \\
& p_{i}:=D\left(\lambda_{i}\right) \quad \text { for } i=2, \cdots, t .
\end{aligned}
$$

Finally, we may assume that $D \mid\left[\varepsilon, \lambda_{2}\right]$ and $D \mid\left[\lambda_{i-1}, \lambda_{i}\right]$ are linear functions $(i=3, \cdots, t)$ (since in the straight-line case each face not in $\left\{I_{1}, \cdots, I_{p}\right\}$ is convex).

Let $h(D)$ be the smallest index $h$ with $2 \leqq h \leqq t-1$ so that the angle between $\overline{p_{h-1} p_{h}}$ and $\overline{p_{h} p_{h+1}}$ is not $180^{\circ}$. If no such $h$ exists, let $h(D):=t$. We may assume that we have chosen $D$ so that (fixing $t=\operatorname{cr}(G, D)) h(D)$ is as large as possible. Let $h:=h(D)$.

First consider the case $h<t$. Choose the largest $\lambda \in[0,1]$ so that the triangle with vertices $p_{1}, p_{h}$, and $p_{h}+\lambda\left(p_{h+1}-p_{h}\right)$ does not intersect $I_{1} \cup \cdots \cup I_{p}$. Let $p_{h}^{\prime}:=p_{h}+$ $\lambda\left(p_{h+1}-p_{h}\right)$. Let $D^{\prime}$ be the piecewise linear function obtained from $D$ by replacing parts $\overline{p_{1} p_{h}}$ and $\overline{p_{h} p_{h}^{\prime}}$ of $D$ by $\overline{p_{1} p_{h}^{\prime}}$.

If $\lambda=1$, then $p_{h}^{\prime}=p_{h+1}$, and hence by (14)(ii) $\overline{p_{1} p_{h}^{\prime}}$ does not intersect any vertex of $G$. So $D^{\prime}$ is a cut, with $\operatorname{cr}\left(G, D^{\prime}\right)=\operatorname{cr}(G, D)$ (by the conditions $(5)$ and (6) for the straight-line case ) and $D^{\prime} \sim D$. As $h\left(D^{\prime}\right)>h(D)$ this contradicts the fact that we have chosen $D$ so that $h(D)$ is as large as possible.

If $\lambda<1$, then $\overline{p_{1} p_{h}^{\prime}}$ intersects a vertex $v$ of $G$, on the boundary of $I_{1} \cup \cdots \cup I_{p}$. This vertex is unique by (14) (ii) and has aperture larger than $180^{\circ}$. Consider a circle $K$ with center $v$, not containing any other vertex of $G$, and not intersecting any edge of $G$ except for those adjacent to $v$. Let $K \backslash\left(I_{1} \cup \cdots \cup I_{p}\right)$ have components $K_{1}, \cdots, K_{h}$. So each $K_{i}$ is a cut. We may assume that $K_{1}$ intersects $D^{\prime}$ twice. So $K_{1}$ is a circular arc of angle larger than $180^{\circ}$. Use the notation $A, B, C, E, F$ for the parts of $D^{\prime}$ and $K_{1}$ as indicated in Fig. 2. Let $H$ denote the part of $D$ from $p_{h}^{\prime}$ to $p_{t}$. As we have chosen $D$ so that (13) is satisfied with $\mathrm{cr}(G, D)$ as small as possible, we have

$$
\begin{aligned}
\operatorname{cr}(G, D)= & \operatorname{cr}(G, E B F H)=\operatorname{cr}(G, E A)+\operatorname{cr}(G, C F H)+\sum_{j=2}^{h} \operatorname{cr}\left(G, K_{j}\right) \\
& +(\text { number of edges terminating at } v) \\
\geqq & \sum_{i=1}^{k} \operatorname{mincr}\left(C_{i}, E A\right)+\sum_{i=1}^{k} \operatorname{mincr}\left(C_{i}, C F H\right)+\sum_{j=2}^{h} \sum_{i=1}^{k} \operatorname{mincr}\left(C_{i}, K_{j}\right) \\
& \left.+\sum_{i=1}^{k} \text { (number of times } v \text { is end point of } C_{i}\right) \geqq \sum_{i=1}^{k} \operatorname{mincr}\left(C_{i}, D\right)
\end{aligned}
$$

(using (6)). This contradicts (13). 


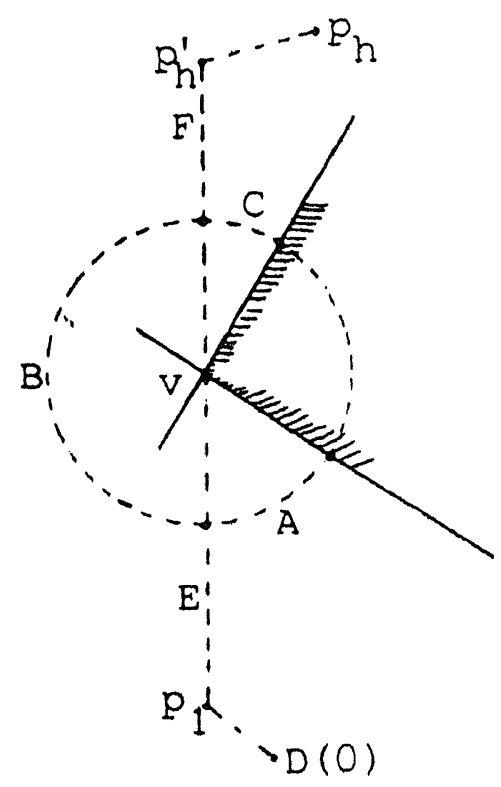

FIG. 2

As $h<t$ leads to a contradiction, we know $h=t$. If the line segment $\overline{D(0) D(1)}$ is not contained in $G$, then by our assumption this line segment forms a straight cut $D^{\prime}$, with $\operatorname{cr}\left(G, D^{\prime}\right)=\operatorname{cr}(G, D)$ and $D^{\prime} \sim D$, whence

$$
\operatorname{cr}(G, D)=\operatorname{cr}\left(G, D^{\prime}\right) \geqq \sum_{i=1}^{k} \operatorname{mincr}\left(C_{i}, D^{\prime}\right)=\sum_{i=1}^{k} \operatorname{mincr}\left(C_{i}, D\right),
$$

contradicting (13). If $\overline{D(0) D(1)}$ is contained in $G$, then $D$ itself forms a straight cut, contradicting (13).

4. Proof of the theorem. We now prove our theorem.

THEOREM. If we are in the straight-line case and the parity condition holds, then there exist pairwise edge-disjoint paths as in (1) if and only if the cut condition holds.

Proof. The proof is by induction on the number of faces not in $\left\{I_{1}, \cdots, I_{p}\right\}$. If each face belongs to $\left\{I_{1}, \cdots, I_{p}\right\}$, then the theorem is trivially true. So assume that not all faces belong to $\left\{I_{1}, \cdots, I_{p}\right\}$.

I. We first consider those situations where the following holds:

$G$ has an edge $e_{0}$, connecting vertices $u$ and $w$, both of degree 2 , so that $e_{0}$ separates a face in $\left\{I_{1}, \cdots, I_{p}\right\}$ from a face not in $\left\{I_{1}, \cdots, I_{p}\right\}$ and so that one of the curves $C_{i}$ connects $u$ and $w$ following $e_{0}$.

Without loss of generality, $e_{0}$ separates face $I_{1}$ from face $F \notin\left\{I_{1}, \cdots, I_{p}\right\}$, and $C_{1}$ connects $u$ and $w$ following $e_{0}$. Moreover, we may assume that none of $C_{2}, \cdots, C_{k}$ passes $e_{0}$ (we can make detours along the other edges of $F$ ). By the parity condition, there exist $h, j$ so that $C_{h}$ has an end point in $u$ and $C_{j}$ has an end point in $w$ (possibly $h=j)$.

Now let $I_{p+1}:=F$. Clearly, $G ; I_{p}, \cdots, I_{p}, I_{p+1} ; C_{1}, \cdots, C_{k}$ is again in the straightline case, in which the parity condition holds. We show

the cut condition holds for $G ; I_{1}, \cdots, I_{p+1} ; C_{1}, \cdots, C_{k}$. 
As the number of faces not in $\left\{I_{1}, \cdots, I_{p+1}\right\}$ is one less than in the original situation, (19) implies by induction that there exist pairwise edge-disjoint paths $P_{1} \sim C_{1}$, $\cdots, P_{k} \sim C_{k}$ in $\mathbb{R}^{2} \backslash\left(I_{1} \cup \cdots \cup I_{p+1}\right)$. This implies $P_{1} \sim C_{1}, \cdots, P_{k} \sim C_{k}$ in $\mathbb{R}^{2} \backslash$ $\left(I_{1} \cup \cdots \cup I_{p}\right)$ as required.

We prove (19). We will refer to $G ; I_{1}, \cdots, I_{p+1} ; C_{1}, \cdots, C_{k}$ as the new structure, and to $G ; I_{1}, \cdots, I_{p} ; C_{1}, \cdots, C_{k}$ as the original structure. For the new structure we use the notation mincr' instead of mincr.

To show (19) by the lemma, it suffices to prove the cut inequality for straight cuts only. Let $D$ be a straight cut in the new structure. If $D(0)$ and $D(1)$ belong to the boundary of $I_{1} \cup \cdots \cup I_{p}$, then $D$ is also a cut in the original structure, and the cut inequality follows $\left(\right.$ as mincr' $\left(C_{i}, D\right)=\operatorname{mincr}\left(C_{i}, D\right)$ for each $\left.i\right)$. If both $D(0)$ and $D(1)$ belong to the boundary of $I_{p+1}=F$, then $\operatorname{mincr}^{\prime}\left(C_{i}, D\right)=0$ for each $i$ (as $F$ is convex), and the cut inequality follows. So we may assume that $D(0)$ belongs to the boundary of $I_{1} \cup \cdots \cup I_{p}$ and $D(1)$ belongs to the boundary of $F$. We can extend $D$ in $\bar{F}$ to a cut $D^{\prime}$ ending on $e_{0}$. Then $D^{\prime}$ is a cut in the original structure. Thus we have

$$
\operatorname{cr}(G, D)=\operatorname{cr}\left(G, D^{\prime}\right)-1 \geqq \sum_{i=1}^{k} \operatorname{mincr}\left(C_{i}, D^{\prime}\right)-1=\sum_{i=1}^{k} \operatorname{mincr}^{\prime}\left(C_{i}, D\right) \text {, }
$$

thus showing the cut inequality for $D$. This proves (19).

II. Now we consider the general case (i.e., we do not assume (18)). As not all faces belong to $\left\{I_{1}, \cdots, I_{p}\right\}$, there exists an edge, say $e_{0}$, separating a face $I_{h}(1 \leqq h \leqq p)$ from a face $F$ not in $\left\{I_{1}, \cdots, I_{p}\right\}$. We may assume $h=1$. Without loss of generality, no path $C_{i}$ intersects $e_{0}$ or $F$ (we can make detours along the boundary of $F$ ). Extend $G$ to a graph $G^{\prime}$ by adding two new vertices, say $u$ and $w$, on $e_{0}$. Let $e_{0}^{\prime}$ be the edge connecting $u$ and $w$. Let $C_{k+1}$ and $C_{k+2}$ be two curves, each connecting $u$ and $w$ via $e_{0}^{\prime}$. We consider two cases.

Case 1. The cut condition holds for $G^{\prime} ; I_{1}, \cdots, I_{p} ; C_{1}, \cdots, C_{k}, C_{k+1}, C_{k+2}$. Now we can apply part I of this proof above, and paths $P_{1}, \cdots, P_{k}, P_{k+1}, P_{k+2}$ as required exist.

Case 2. The cut condition does not hold for $G^{\prime} ; I_{1}, \cdots, I_{p} ; C_{1}, \cdots, C_{k}, C_{k+1}$, $C_{k+2}$. Since also in this new situation we are in the straight-line case, by the lemma there exists a straight cut $D$ so that

$$
\operatorname{cr}\left(G^{\prime}, D\right)<\sum_{i=1}^{k+2} \operatorname{mincr}\left(C_{i}, D\right)
$$

Since mincr $\left(C_{k+1}, D\right)=\operatorname{mincr}\left(C_{k+2}, D\right) \leqq 1$ and since the parity condition holds for $G ; I_{1}, \cdots, I_{p} ; C_{1}, \cdots, C_{k}$ we know

$$
\operatorname{cr}(G, D)=\sum_{i=1}^{k} \operatorname{mincr}\left(C_{i}, D\right),
$$

and $\operatorname{mincr}\left(C_{k+1}, D\right)=\operatorname{mincr}\left(C_{k+2}\right)=1$. Hence $D$ has one of its end points on $e_{0}^{\prime}$.

As the cut condition holds for $G ; I_{1}, \cdots, I_{p} ; C_{1}, \cdots, C_{k}$, there exists a "fractional" packing of paths $P_{1}^{1}, \cdots, P_{1}^{t_{1}}, \cdots, P_{k}^{1}, \cdots, P_{k}^{t_{k}}$, with coefficients $\lambda_{1}^{1}, \cdots, \lambda_{1}^{t_{1}}, \cdots$, $\lambda_{k}^{1}, \cdots, \lambda_{k}^{t_{k}}>0$, satisfying (7). By (22), at least one of the $P_{i}^{j}$, say $P_{1}^{1}$, passes edge $e_{0}$. So $P_{1}^{1}=R_{1} e_{0}^{\prime} R_{2}$ for certain paths $R_{1}$ and $R_{2}$.

We now show the following claim.

ClaIM. For each straight cut $D^{\prime}$ ( for $\left.G^{\prime}\right)$ we have

$$
\operatorname{mincr}\left(R_{1}, D^{\prime}\right)+\operatorname{mincr}\left(C_{k+1}, D^{\prime}\right)+\operatorname{mincr}\left(R_{2}, D^{\prime}\right) \leqq \operatorname{mincr}\left(C_{1}, D^{\prime}\right)+2 \text {. }
$$


Proof of the claim. Since

$$
\operatorname{cr}(G, D)=\sum_{i=1}^{k} \operatorname{mincr}\left(C_{i}, D\right) \leqq \sum_{i=1}^{k} \sum_{j=1}^{t_{i}} \lambda_{i}^{j} \cdot \operatorname{cr}\left(P_{i}^{j}, D\right) \leqq \operatorname{cr}(G, D),
$$

and since $\lambda_{1}^{1}>0$, we know that $\operatorname{cr}\left(P_{1}^{1}, D\right)=\operatorname{mincr}\left(C_{1}, D\right)$.

Without loss of generality, $\left(\left.P_{1}^{1}\right|_{0} ^{1 / 4}\right)$ coincides with path $R_{1},\left(\left.P_{1}^{1}\right|_{1 / 4} ^{3 / 4}\right)$ with $C_{k+1}$, and $\left(P_{1}^{1} \mid \frac{1}{3 / 4}\right)$ with $R_{2}$. Moreover, we may assume that $P_{1}^{1}(1 / 2)=D(0)$.

Let $D^{\prime}$ be any straight cut. To show (23) we may assume that $D$ and $D^{\prime}$ intersect each other at most once, and that if $D^{\prime}$ intersects $e_{0}^{\prime}$, then $D$ and $D^{\prime}$ do not intersect.

Let

$$
X:=\left\{(x, y) \in[0,1] \times[0,1] \mid P_{1}^{1}(x)=D^{\prime}(y)\right\} .
$$

Let $\approx$ be as in $(10)$. So mincr $\left(C_{1}, D^{\prime}\right)$ is equal to the number of odd classes of $\approx$. We show

if $(x, y),\left(x^{\prime}, y^{\prime}\right),\left(x^{\prime \prime}, y^{\prime \prime}\right),\left(x^{\prime \prime \prime}, y^{\prime \prime \prime}\right) \in X$ so that $(x, y) \approx\left(x^{\prime}, y^{\prime}\right),\left(x^{\prime \prime}, y^{\prime \prime}\right) \approx$ $\left(x^{\prime \prime \prime}, y^{\prime \prime \prime}\right), x, x^{\prime \prime} \in\left(0, \frac{1}{2}\right)$ and $x^{\prime}, x^{\prime \prime \prime} \in\left(\frac{1}{2}, 1\right)$, then $D$ and $D^{\prime}$ intersect and $(x, y) \approx\left(x^{\prime \prime}, y^{\prime \prime}\right)$.

Indeed, as $(x, y) \approx\left(x^{\prime}, y^{\prime}\right)$, we know $\left(P_{1}^{1}||_{x}^{x^{\prime}}\right) \sim\left(D^{\prime} \mid \begin{array}{l}y^{\prime} \\ y\end{array}\right)$. So $\left(P_{1}^{1} \mid \begin{array}{l}x^{\prime} \\ x\end{array}\right)\left(\left.D^{\prime}\right|_{y^{\prime}} ^{y}\right)$ forms a homotopically trivial cycle $K$. Since $\left(P_{1}^{1} \mid \begin{array}{l}x^{\prime} \\ x\end{array}\right)$ passes $D(0), D$ splits $K$ into two homotopically trivial cycles. That is, there is a $\lambda \in(0,1]$ so that

$$
\text { either (i) } \exists z \in\left[x, x^{\prime}\right]:\left(\left.P_{1}^{1}\right|_{z} ^{1 / 2}\right)\left(\left.D\right|_{0} ^{\lambda}\right) \text { is a homotopically trivial cycle, }
$$

or (ii) $\exists z \in\left(y, y^{\prime}\right):\left(\left.P_{1}^{1}\right|_{x} ^{1 / 2}\right)\left(\left.D\right|_{1 / 2} ^{\lambda}\right)\left(\left.D^{\prime}\right|_{z} ^{y}\right)$ is a homotopically trivial cycle.

Since $\operatorname{cr}\left(P_{1}^{1}, D\right)=\operatorname{mincr}\left(P_{1}^{1}, D\right),(27)$ (i) does not occur. So (27) (ii) applies. Hence

$$
\left(\left.P_{1}^{1}\right|_{x} ^{1 / 2}\right) \sim\left(\left.D^{\prime}\right|_{y} ^{z}\right)\left(\left.D\right|_{\lambda} ^{1 / 2}\right)
$$

In particular, $D$ and $D^{\prime}$ intersect, with $D(\lambda)=D^{\prime}(z)$. We similarly derive from the fact that $\left(x^{\prime \prime}, y^{\prime \prime}\right) \approx\left(x^{\prime \prime \prime}, y^{\prime \prime \prime}\right)$ that

$$
\left(\left.P_{1}^{1}\right|_{x^{\prime \prime}} ^{1 / 2}\right) \sim\left(\left.D^{\prime}\right|_{y^{\prime \prime}} ^{2}\right)\left(\left.D\right|_{\lambda} ^{1 / 2}\right)
$$

Therefore,

$$
\left(\left.P_{1}^{1}\right|_{x} ^{x^{\prime \prime}}\right) \sim\left(\left.P_{1}^{1}\right|_{x} ^{1 / 2}\right)\left(\left.P_{1}^{1}\right|_{1 / 2} ^{x^{\prime \prime}}\right) \sim\left(\left.D^{\prime}\right|_{y} ^{z}\right)\left(\left.D\right|_{\lambda} ^{1 / 2}\right)\left(\left.D\right|_{1 / 2} ^{\lambda}\right)\left(\left.D^{\prime}\right|_{z} ^{y^{\prime \prime}}\right) \sim\left(\left.D^{\prime}\right|_{y} ^{y^{\prime \prime}}\right) .
$$

So $(x, y) \approx\left(x^{\prime \prime}, y^{\prime \prime}\right)$. This shows $(26)$.

Now $\operatorname{cr}\left(C_{k+1}, D^{\prime}\right) \leqq 1$. If $\operatorname{cr}\left(C_{k+1}, D^{\prime}\right)=0$, then the above implies

$$
\text { odd }\left(P_{1}^{1}, D^{\prime}\right) \geqq\left(\operatorname{odd}\left(R_{1}, D^{\prime}\right)-1\right)+\left(\operatorname{odd}\left(R_{2}, D^{\prime}\right)-1\right),
$$

since by (26) all but at most one class of intersections of $R_{1}$ and $D^{\prime}$ is also a class of intersections of $P_{1}^{1}$ and $D^{\prime}$. Similarly for $R_{2}$. Equation (31) implies (23).

If $\operatorname{cr}\left(C_{k+1}, D^{\prime}\right)=1$, then $D$ and $D^{\prime}$ do not intersect, by assumption. Hence, by (26), no class of intersections of $P_{1}^{1}$ and $D^{\prime}$ contains both $(x, y)$ and $\left(x^{\prime}, y^{\prime}\right)$ with $x \in$ $\left(0, \frac{1}{2}\right)$ and $x^{\prime} \in\left(\frac{1}{2}, 1\right)$. Since $\operatorname{cr}\left(C_{k+1}, D^{\prime}\right)=1$, there is only one element $(x, y)$ in $X$ with $x \in\left(\frac{1}{4}, \frac{3}{4}\right)$. Except for the class of intersections of $P_{1}^{1}$ and $D^{\prime}$ containing this element, all other classes also form a class of intersections of $R_{1}$ and $D^{\prime}$ or of $R_{2}$ and $D^{\prime}$. Hence

$$
\text { odd }\left(P_{1}^{1}, D^{\prime}\right) \geqq \operatorname{odd}\left(R_{1}, D^{\prime}\right)+\operatorname{odd}\left(R_{2}, D^{\prime}\right)-1 \text {, }
$$

and (23) follows. 
We next show

$$
\text { the cut condition holds for } G^{\prime} ; I_{1}, \cdots, I_{p} ; R_{1}, R_{2}, C_{2}, \cdots, C_{k}, C_{k+1} \text {. }
$$

Suppose not. Since we are again in the straight-line case, by the lemma there exists a straight cut $D^{\prime}$ so that

$$
\operatorname{mincr}\left(R_{1}, D^{\prime}\right)+\operatorname{mincr}\left(R_{2}, D^{\prime}\right)+\sum_{i=2}^{k+1} \operatorname{mincr}\left(C_{i}, D^{\prime}\right) \geqq \operatorname{cr}\left(G, D^{\prime}\right)+2,
$$

using the fact that the parity condition holds also for $G^{\prime} ; I_{1}, \cdots, I_{p} ; R_{1}, R_{2}, C_{2}, \cdots$, $C_{k+1}$. Since the cut condition does hold for $G^{\prime} ; I_{1}, \cdots, I_{p} ; C_{1}, \cdots, C_{k}$, it follows that

$$
\operatorname{mincr}\left(R_{1}, D^{\prime}\right)+\operatorname{mincr}\left(R_{2}, D^{\prime}\right)+\operatorname{mincr}\left(C_{k+1}, D^{\prime}\right)>\operatorname{mincr}\left(C_{1}, D^{\prime}\right) \text {. }
$$

Hence

$$
\operatorname{cr}\left(P_{1}^{1}, D^{\prime}\right)=\operatorname{cr}\left(R_{1}, D^{\prime}\right)+\operatorname{cr}\left(R_{2}, D^{\prime}\right)+\operatorname{cr}\left(C_{k+1}, D^{\prime}\right)>\operatorname{mincr}\left(C_{1}, D^{\prime}\right) .
$$

Therefore,

$$
\begin{aligned}
\operatorname{cr}\left(G, D^{\prime}\right) & \geqq \sum_{i=1}^{k} \sum_{j=1}^{t_{i}} \lambda_{i}^{j} \cdot \operatorname{cr}\left(P_{i}^{j}, D^{\prime}\right)>\sum_{i=1}^{k} \sum_{j=1}^{t_{i}} \lambda_{i}^{j} \cdot \operatorname{mincr}\left(C_{i}, D^{\prime}\right) \\
& =\sum_{i=1}^{k} \operatorname{mincr}\left(C_{i}, D^{\prime}\right) .
\end{aligned}
$$

However, (34) and (37) imply

$$
\begin{gathered}
\operatorname{mincr}\left(R_{1}, D^{\prime}\right)+\operatorname{mincr}\left(R_{2}, D^{\prime}\right)+\sum_{i=2}^{k+1} \operatorname{mincr}\left(C_{i}, D^{\prime}\right) \geqq \operatorname{cr}\left(G, D^{\prime}\right)+2 \\
>\sum_{i=1}^{k} \operatorname{mincr}\left(C_{i}, D^{\prime}\right)+2,
\end{gathered}
$$

contradicting the claim.

So (33) holds, and hence by part I of this proof there exist pairwise edge-disjoint paths $Q_{1}^{\prime} \sim R_{1}, Q_{1}^{\prime \prime} \sim R_{2}, Q_{2} \sim C_{2}, \cdots, Q_{k} \sim C_{k}, Q_{k+1} \sim C_{k+1}$. By sticking $Q_{1}^{\prime}$, $Q_{k+1}, Q_{1}^{\prime \prime}$ to one path, which is homotopic to $C_{1}$, we obtain paths as required.

\section{REFERENCES}

[1] C. VAN HOESEl AND A. SCHRIJVE, Edge-disjoint homotopic paths in a planar graph with one hole, J. Combin. Theory Ser. B, 48 (1990), pp. 77-91.

[2] M. Kaufmann AND K. Mehlhorn, On local routing of two-terminal nets, J. Combin. Theory Ser. B, to appear.

[3] H. OKamura AND P. D. Seymour, Multicommodity flows in planar graphs, J. Combin. Theory Ser. B, 31 (1981), pp. 75-81.

[4] A. SCHRIJVer, Decomposition of graphs on surfaces and a homotopic circulation theorem, J. Combin. Theory Ser. B, to appear. 